\title{
Angular correlation in the two-electron continuum
}

\author{
A. S. Kheifets* \\ Research School of Physical Sciences, The Australian National University, Canberra ACT 0200, Australia \\ I. Bray $^{\dagger}$ \\ Center for Atomic, Molecular, and Surface Physics, Physics and Energy Studies, Murdoch University, Perth 6150, Australia
}

(Received 11 August 2005; published 28 February 2006)

\begin{abstract}
Following absorption of a single photon, angles of simultaneous emission of two electrons from a $\operatorname{He}\left(n^{1} S\right)$ atom become more correlated with increasing $n$. We find that the strength of this correlation is due to the two-electron continuum of the electron-impact ionization of the $\mathrm{He}^{+}(n s)$ ion. The strength is determined by the width of the momentum profile of the ionic $n s$ state but not the strength of the electron correlation in the $\mathrm{He}$ initial state. This can explain the increasing (over He) angular correlation strength found in double photoionization of targets such as $\mathrm{Be}, \mathrm{Ne}$, and $\mathrm{H}_{2}$.
\end{abstract}

DOI: 10.1103/PhysRevA.73.020708

PACS number(s): $32.80 . \mathrm{Fb}, 32.80 . \mathrm{Rm}, 42.50 . \mathrm{Hz}$

Many-electron correlations in atoms have been a subject of intense scrutiny for many decades. The many-electron correlation in a bound atomic state can be understood as a deviation from predictions of the Hartree-Fock self-consistent field model which is the best theoretical description still compatible with a notion of independent electrons. Manyelectron correlations in unbound atomic states with one or several electrons in continuum elude a simple definition. Broadly, this concept can be applied to the processes driven strongly by interelectron interaction and thus deviating from predictions of the lowest-order perturbation theories. Presently, these processes, collectively termed as correlated many-body quantum dynamics, are the focus of particular interest with implications spanning physics, chemistry, and biology alike. Fundamental processes like double photoionization (DPI), especially in the $\mathrm{He}$ atom, play a central, benchmarking role in this context. Understanding the role of interelectron interaction in this seemingly simple process, involving two electrons, provides insights to electron correlations in more complex breakup processes in many-electron systems.

Since the pioneering work of Wannier dating back to more than 50 years ago [1], it has been established that the two-electron breakup is a tightly correlated process with the back-to-back emission being the only possible route to double ionization at the threshold. At a small excess energy $E$ above the threshold, the electron pair acquires some dynamical freedom and can deviate from the back-toback emission $\theta_{12}=\pi$ within a Gaussian distribution $\exp \left[-2 \ln 2\left(\pi-\theta_{12}\right)^{2} / \Delta \theta_{12}^{2}\right]$ with a finite angular correlation width $\Delta \theta_{12} \propto E^{1 / 4}[2,3]$. This result is based on the solution of the two-electron Schrödinger equation in the so-called Coulomb zone where the potential energy dominates over the kinetic energy and the two-electron escape follows closely the Wannier ridge $r_{1} \simeq r_{2}$ and $\theta_{12} \simeq \pi$. At a sufficiently large separation $R=\left(r_{1}^{2}+r_{2}^{2}\right)^{1 / 2}$, in the "far zone," the kinetic energy

\footnotetext{
*Electronic address: A.Kheifets@anu.edu.au

†Electronic address: I.Bray@murdoch.edu.au
}

becomes dominant and the Coulomb force is not able to return the electrons back to the nucleus. The angular correlation width in the far zone remains unchanged until the electrons reach the detectors and it is this correlation width that determines the experimentally measured fully differential cross section. At small separations $R$, the Coulomb zone borders with the "interaction zone." In this zone, details of the interaction of the two-electron system with the external field become important. These details, however, are completely ignored in the Wannier theory since they do not affect the threshold law.

In this Rapid Communication we analyze the processes in the interaction zone from the point of view of their effect on the strength (size of $\Delta \theta_{12}$ ) of the angular correlation in DPI. In particular, we are motivated by trying to understand the narrowing of the DPI angular correlation width in Be $[4,5]$, $\mathrm{Ne}$ [6], and $\mathrm{H}_{2}$ [7-9]. Previously, this narrowing was attributed to stronger electron-electron correlations in the valence $2 s^{2}$ shell of Be than in the $1 s^{2}$ shell of He (see abstract of Ref. [5]). We offer an alternative explanation to this phenomenon. As a case study, without too much loss of generality, we consider the He atom in a range of initial $n{ }^{1} S$ states for $n \leqslant 3$. For $n=1$ we have the extreme of a highly correlated initial state. For $n=3$ we have a rather diffuse initial state. We expect that any systematic, as a function of $n$, behavior here will translate well to more complex targets.

Near the threshold, the DPI proceeds primarily via one electron absorbing the photon and then ionizing the residual ion via collisional impact. A competing "shake-off" process is relatively inefficient near the threshold. In this process, the second electron is shaken off into continuum due to a sudden change of the atomic potential after departure of the first photoelectron. If this photoelectron is slow, the remaining target electron has sufficient time to adjust its state to a changing environment and remains bound in the ion. The shake-off process is greatly assisted by the ground-state correlation which can be thought of as a virtual excitation of a target electron pair. If one electron is ionized during this excitation, the second electron has a much greater chance to be shaken off into continuum. Insensitivity of DPI to the ground-state correlation near the threshold was demonstrated 
in Ref. [10] where a range of ground-state wave functions with a varying amount of correlation produced essentially the same DPI cross section in all gauges of electromagnetic interaction. This is another indication that the shake-off process is switched off near the threshold.

The DPI process is most probable if the photon is absorbed by an "inner" electron which then ejects an "outer" one. In this configuration, the recoil momentum is most readily accommodated by the nucleus [11]. At a small excess energy, the "outer" electron has sufficient time to adjust itself to a slow departure of the inner electron and therefore remains in the $n s$ ionic state. Thus, in the case of the $\operatorname{He}\left(n^{1} S\right)$ target the DPI process, after photon absorption, proceeds via electron-impact ionization of the corresponding $\mathrm{He}^{+}(n s)$ ion. We may calculate $\Delta \theta_{12}$ explicitly for the $e-\mathrm{He}^{+}(n s)$ system. If there is a strong similarity with the $\Delta \theta_{12}$ of the DPI then we can exclude the electron-electron correlations in the $\mathrm{He}$ initial $n{ }^{1} S$ state from contributing to the $\Delta \theta_{12}$ of the DPI.

We perform numerical calculations of the angular correlation width in the two-electron continuum using the convergent close-coupling (CCC) method. The DPI calculations have been described earlier $[12,13]$. The only difference introduced here is the use of the box-space basis states [14] which allowed us to approach the double ionization threshold to within $0.4 \mathrm{eV}$. The derivation of the angular correlation width for the electron-impact ionization is similar to DPI. We write the amplitude of the electron impact ionization of the target state $i$ in the form of a partial wave expansion [15],

$$
\begin{aligned}
f_{i}^{S}\left(\boldsymbol{k}_{1}, \boldsymbol{k}_{2}\right)= & \frac{1}{\sqrt{4 \pi}} \frac{1}{\hat{l}_{i}} \sqrt{\frac{k_{0}}{k_{1}}} \sum_{l_{0} l_{1} l_{2} J}\left(2 l_{0}+1\right)^{1 / 2} \\
& \times C_{l_{0} l_{i} m_{i}}^{J m_{i}} T_{l_{0} l_{1} l_{2}}^{J S}\left(k_{1}, k_{2}\right) \mathcal{Y}_{J m_{i}}^{l_{1} l_{2}}\left(\boldsymbol{k}_{1}, \boldsymbol{k}_{2}\right) .
\end{aligned}
$$

Here, the projectile of momentum $\boldsymbol{k}_{0}$ is directed along the quantization $z$ axis and the total spin of the scattering system is $S$. The $T$ matrix entering Eq. (1) is obtained by the projection of the true continuum state $\boldsymbol{k}_{2}$ onto the same-energy pseudostate,

$$
\begin{aligned}
T_{l_{0} l_{1} l_{2}}^{J S}\left(k_{1}, k_{2}\right)= & (-i)^{l_{0}+l_{1}+l_{2}} e^{i\left(\delta_{l_{0}}+\delta_{l_{1}}+\delta_{l_{2}}\right)}\left\langle k_{2} l_{2} \| n l_{2}\right\rangle \\
& \times\left\langle k_{1} \phi_{n}\left\|T^{J S}\right\| \phi_{i} k_{0}\right\rangle .
\end{aligned}
$$

Here $\left\langle k_{1} \phi_{n}\left\|T^{J S}\right\| \phi_{i} k_{0}\right\rangle$ is the bare $T$ matrix stripped of all phase factors and angular momentum projections.

We shall restrict ourselves to the dipole singlet amplitude which is the only amplitude relevant to DPI. In this case for an $s$ target state $l_{i}=0, l_{0}=1, J=1, S=0$ and Eq. (1) can be further simplified,

$$
f_{s p}\left(\boldsymbol{k}_{1}, \boldsymbol{k}_{2}\right)=\sqrt{\frac{3}{4 \pi}} \sqrt{\frac{k_{0}}{k_{1}}} \sum_{l_{1} l_{2}} T_{l_{1} l_{2}}\left(k_{1}, k_{2}\right) \mathcal{Y}_{10}^{l_{1} l_{2}}\left(\boldsymbol{k}_{1}, \boldsymbol{k}_{2}\right),
$$

where $l_{2}=l_{1} \pm 1$. The $(e, 2 e)$ cross section is given by the squared amplitude (3)

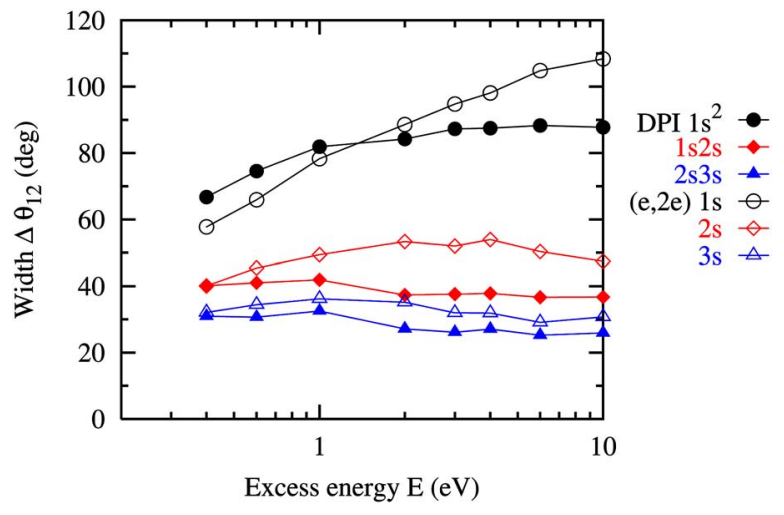

FIG. 1. (Color online) Gaussian width parameters for DPI of $\mathrm{He}\left(n^{1} S\right)$ and $(e, 2 e)$ of $\mathrm{He}^{+}(n s)$ for $n \leqslant 3$ plotted versus excess energy over the corresponding double ionization threshold. Circles, diamonds, and triangles correspond to widths for the $n=1,2$, and 3 initial states, respectively. Filled symbols are for DPI on $\mathrm{He}$ and open symbols are for $(e, 2 e)$ on $\mathrm{He}^{+}$.

$$
\frac{d \sigma_{s p}}{d \Omega_{1} d \Omega_{2} d E_{2}}=(2 \pi)^{4} \frac{k_{1} k_{2}}{k_{0}}\left|f_{s p}\left(\boldsymbol{k}_{1}, \boldsymbol{k}_{2}\right)\right|^{2} .
$$

After we introduce symmetric and antisymmetric combinations of the $T$ matrices,

$$
T_{l_{1} l_{2}}^{ \pm}\left(E_{1}, E_{2}\right)=\frac{1}{2}\left\{T_{l_{1} l_{2}}\left(E_{1}, E_{2}\right) \pm T_{l_{1} l_{2}}\left(E_{2}, E_{1}\right)\right\}
$$

the angular momentum summation in (3) can be reduced to the sum over a single variable. This allows us to parametrize the cross section (3) in the form,

$$
\frac{d \sigma_{s p}}{d \Omega_{1} d \Omega_{2} d E_{2}} \propto\left|\left(k_{1 z}+k_{2 z}\right) g^{+}\left(E_{1}, E_{2}\right)+\left(k_{1 z}-k_{2 z}\right) g^{-}\left(E_{1}, E_{2}\right)\right|^{2},
$$

where the symmetric and antisymmetric amplitudes are

$$
\begin{aligned}
g^{ \pm}\left(E_{1}, E_{2}\right)= & \frac{1}{4 \pi} \sum_{l=0}^{\infty} \frac{(-1)^{l}}{\sqrt{l+1}}\left[P_{l+1}^{\prime}\left(\cos \theta_{12}\right) \mp P_{l}^{\prime}\left(\cos \theta_{12}\right)\right] \\
& \times T_{l l+1}^{ \pm}\left(E_{1}, E_{2}\right) .
\end{aligned}
$$

Here we used expressions for the bipolar harmonics $\mathcal{Y}_{10}^{l_{1} l_{2}}$ due to Manakov et al. [16]. The mutual electron angle is defined by $\cos \theta_{12}=\hat{k}_{1} \cdot \hat{k}_{2}$. We note that Eq. (7) is identical to Eq. (13) of Ref. [13] except for the $T$-matrix elements which are substituted by the dipole matrix elements in the DPI case.

We concentrate on the special case of equal energy sharing when $g^{-}$vanishes. Close to double ionization threshold, the symmetric amplitude can be fitted with the Gaussian ansatz,

$$
\left|g^{+}\right|=A \exp \left[-2 \ln 2 \frac{\left(\pi-\theta_{12}\right)^{2}}{\Delta \theta_{12}^{2}}\right],
$$

where the Gaussian width parameter $\Delta \theta_{12}$ is the measure of strength of the angular correlation.

In Fig. 1, we plot the Gaussian width parameters for DPI of the He atom in the $n^{1} S$ initial states, where $n \leqslant 3$, as 


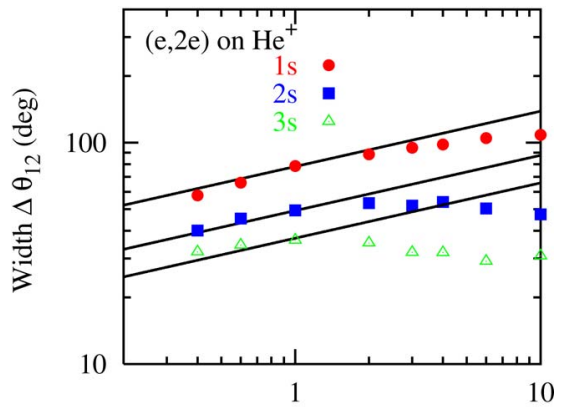

(a) Excess energy $\mathrm{E}(\mathrm{eV})$

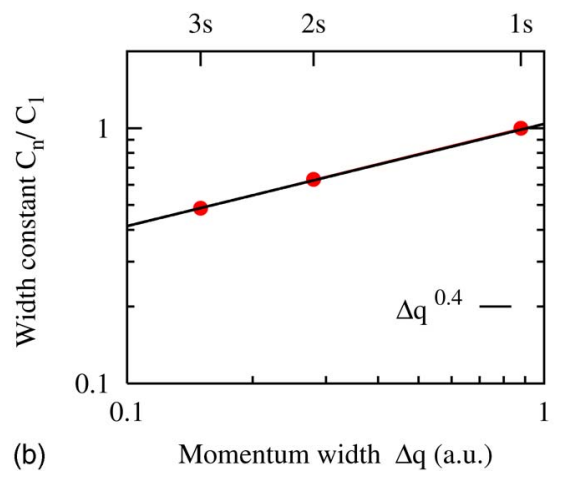

FIG. 2. (Color online) Top panel: Gaussian width parameters for the $(e, 2 e)$ on $\mathrm{He}^{+}$ion in various $n s$ states as functions of excess energy over the corresponding ionization threshold. Solid lines indicate the fitting with Eq. (9). Bottom panel: Energy-independent width constants $C_{n}$ in Eq. (9) plotted versus the width of the corresponding momentum profile extracted from Fig. 3.

functions of the excess energy over the corresponding double ionization threshold. In the same figure, we overplot the Gaussian width parameters for the electron impact ionization of the $\mathrm{He}^{+}$ion in the corresponding $n s$ state.

We observe that there is a systematic reduction in the widths as $n$ increases. The angular correlation widths of the two processes follow each other closely and, for $n=2$ and 3 , merge near the threshold. This indicates that the angular correlation width in DPI is indeed dominated by the corresponding $(e, 2 e)$ process. We fit the energy-dependent angular correlation width of the latter process by the Wannier threshold law

$$
\Delta \theta_{12}(E)=C_{n} E^{1 / 4}
$$

where $C_{n}$ is the angular correlation width at $1 \mathrm{eV}$ excess energy and $E$ is measured in $\mathrm{eV}$. This fitting procedure is exhibited graphically on the top panel of Fig. 2. The energyindependent preexponential factors $C_{n}$ are found to be $78^{\circ}$, $49^{\circ}$, and $38^{\circ}$ for the $n=1,2$, and 3 states of $\mathrm{He}^{+}$, respectively.

We now demonstrate that the factor $C_{n}$ is strongly related to the width of the momentum profile [squared momentumspace wave function $\left.\left|R_{n s}(q)\right|^{2}\right]$ of the target ion state being ionized. An analogy to this fact can be drawn from the binary $(e, 2 e)$ reaction in which the width of the angular distribution of the ejected electron is determined by the momentum profile of the target orbital $[17,18]$. We plot these profiles in Fig. 3. For better visibility, we normalize all the profiles to that of

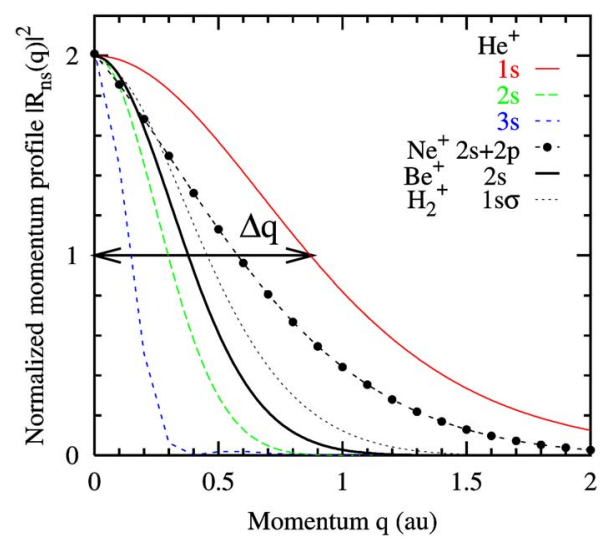

FIG. 3. (Color online) Momentum profiles (squared momentum space wave functions) $\left|R_{n s}(q)\right|^{2}$ of the lowest $n s$ states of the $\mathrm{He}^{+}$ ion, the mixed $2 s$ plus $2 p$ state of $\mathrm{Ne}^{+}$, the $2 s$ state of the $\mathrm{Li}^{+}$ion, and the $1 s \sigma$ state of the $\mathrm{H}_{2}^{+}$ion. The momentum profiles are normalized to $\left|R_{1 s}\right|^{2}$ of $\mathrm{He}^{+}$at its maximum.

the ground-state $1 s$ orbital of $\mathrm{He}^{+}$ion. We see that the range of the possible momenta available in the bound state is rapidly decreasing from $1 s$ to $3 s$. Therefore, the total momentum of the two electrons after an ionizing collision, which determines the range of mutual angles in the interaction zone, will have the largest spread following ionization of the $1 s$ ground state. We illustrate this dependence in Fig. 3, where we plot the energy-independent preexponential factor $C_{n}$ in Eq. (9) versus the width of the momentum profile at half maximum $\Delta q$ of the corresponding $\mathrm{H}^{+}$ion state $n s$ extracted from Fig. 3. We see a clear dependence which can be approximated by a power law $C_{n} / C_{1} \propto \Delta q^{0.4}$.

Momentum profiles of other singly ionized targets $\mathrm{Ne}^{+}$, $\mathrm{Li}^{+}$, and $\mathrm{H}_{2}{ }^{+}$are also plotted in Fig. 3. In terms of the width of the corresponding momentum profile, these targets fall between the $\mathrm{He}^{+} 1 s$ and $2 s$ states. We did not perform the electron impact ionization calculations on targets other than $\mathrm{He}^{+}$and cannot map these targets in the bottom panel of Fig. 2. However, we can compare the relative strength of the angular correlation in DPI of $\mathrm{Ne}, \mathrm{Li}$, and $\mathrm{H}_{2}$ with that of the ground-state $\mathrm{He}$ at the same excess energy. A welldocumented case is $10+10 \mathrm{eV}$ energy sharing. In this case, $\Delta \theta_{12}$ is $91^{\circ}$ for $\mathrm{He}$ and $78^{\circ}$ for $\mathrm{H}_{2}[7,8], 68^{\circ}$ for $\mathrm{Be}[4,5]$, and $74^{\circ}$ in $\mathrm{Ne}$ [6]. If we place these targets in increasing order of $\Delta \theta_{12}$, we find a direct correspondence with the width of the momentum profiles exhibited in Fig. 3 except for the close width values of $\mathrm{Ne}$ and $\mathrm{H}_{2}$ which are swapped. The $\mathrm{Ne}$ atom represents a special case of a strong intershell correlation which affects both the single and double photoionization [19]. Strictly speaking, this is not a ground-state effect. However, it can be incorporated into DPI calculations through an empirical mixing of the $2 s$ and $2 p$ orbitals [6]. Without this mixing, the width of the momentum profile of the pure $2 s$ orbital in $\mathrm{Ne}^{+}$is almost the same as $1 s$ in $\mathrm{He}^{+}$. So is the angular correlation within DPI.

From these findings we conclude that the strength of the angular correlation in DPI comes primarily from the momentum distribution of the corresponding orbital of the singly ionized target. More specifically, the angular correlation width in DPI is a result of interplay of two distinct processes. 
It is initially set up in the interaction region during an ionizing collision of the photoelectron with the positively charged ion. This ionizing collision creates an electron pair with the spread of mutual angles determined by the momentum profile of the target electron bound in the ion. Then the electron pair travels through the Coulomb zone which greatly reduces the spread of the mutual angles focusing them around $180^{\circ}$. As a result, the electron pair emerges in the far zone with the angular correlation width which depends on the size of the Coulomb zone [energy dependence $E^{1 / 4}$ in Eq. (9)] and the momentum profile of the bound ion state (energyindependent preexponential factor $C_{n}$ ).

According to this scenario, at the same excess energy above the double ionization threshold, the $\mathrm{He}$ atom in the ground state will have the largest angular correlation width of DPI due to the largest width of the momentum profile of the $1 s$ state of the bare $Z=2$ Coulomb center. All other neutral atomic and molecular targets, after single ionization, will have lesser bound states either due to screening by other target electrons in atoms or a delocalized Coulomb center in molecules. Therefore, these atomic and molecular targets will have smaller correlation widths of the DPI than say for He initially in the ground state. This fact is not related to the amount of the ground-state correlation in the neutral target. It is the properties of the singly charged ion that determine the angular correlation width.

The authors wish to thank Australian Partnership for Advanced Computing (APAC) and ISA Technologies, Perth, Western Australia, for provision of their computing facilities. Support of the Australian Research Council in the form of Discovery Grant No. DP0451211 is acknowledged.
[1] G. H. Wannier, Phys. Rev. 90, 817 (1953).

[2] A. R. P. Rau, J. Phys. B 9, L283 (1976).

[3] J. M. Feagin, J. Phys. B 17, 2433 (1984).

[4] A. S. Kheifets and I. Bray, Phys. Rev. A 65, 012710 (2002).

[5] F. Citrini, L. Malegat, P. Selles, and A. K. Kazansky, Phys. Rev. A 67, 042709 (2003).

[6] P. Bolognesi, R. Flammini, A. Kheifets, I. Bray, and L. Avaldi, Phys. Rev. A 70, 062715 (2004).

[7] T. J. Reddish, J. P. Wightman, M. A. MacDonald, and S. Cvejanović, Phys. Rev. Lett. 79, 2438 (1997).

[8] J. P. Wightman, S. Cvejanović, and T. J. Reddish, J. Phys. B 31, 1753 (1998).

[9] T. Weber et al., Phys. Rev. Lett. 92, 163001 (2004).

[10] A. S. Kheifets and I. Bray, Phys. Rev. A 57, 2590 (1998).
[11] M. Y. Amusia, E. G. Drukarev, V. G. Gorshkov, and M. O. Kazachkov, J. Phys. B 8, 1248 (1975).

[12] A. S. Kheifets and I. Bray, Phys. Rev. A 62, 065402 (2000).

[13] A. S. Kheifets and I. Bray, Phys. Rev. A 65, 022708 (2002).

[14] I. Bray, K. Bartschat, and A. T. Stelbovics, Phys. Rev. A 67, 060704(R) (2003).

[15] I. Bray and D. V. Fursa, Phys. Rev. A 54, 2991 (1996).

[16] N. L. Manakov, S. I. Marmo, and A. V. Meremianin, J. Phys. B 29, 2711 (1996).

[17] A. E. Glassgold and G. Ialongo, Phys. Rev. 175151 (1968).

[18] M. E. Rudd, Phys. Rev. A 44, 1644 (1991).

[19] M. Y. Amusia, Atomic Photoeffect (Plenum Press, New York, 1990). 